\title{
fMRI Studies of Stroop Tasks Reveal Unique Roles of Anterior and Posterior Brain Systems in Attentional Selection
}

\author{
Marie T. Banich, Michael P. Milham, Ruthann Atchley, Neal J. Cohen, \\ Andrew Webb, Tracey Wszalek, Arthur F. Kramer, Zhei-Pei Liang, \\ Alexander Wright, Joel Shenker, and Richard Magin
}

The Beckman Institute, University of Illinois at Urbana-Champaign

\begin{abstract}
The brain's attentional system identifies and selects information that is task-relevant while ignoring information that is task-irrelevant. In two experiments using functional magnetic resonance imaging, we examined the effects of varying task-relevant information compared to task-irrelevant information. In the first experiment, we compared patterns of activation as attentional demands were increased for two Stroop tasks that differed in the task-relevant information, but not the task-irrelevant information: a color-word task and a spatial-word task. Distinct subdivisions of dorsolateral prefrontal cortex and the precuneus became activated for each task, indicating differential sensitivity of these regions to taskrelevant information (e.g., spatial information vs. color). In the second experiment, we compared patterns of activation with increased attentional demands for two Stroop tasks that differed in task-irrelevant information, but not task-relevant
\end{abstract}

information: a color-word task and color-object task. Little differentiation in activation for dorsolateral prefrontal and precuneus regions was observed, indicating a relative insensitivity of these regions to task-irrelevant information. However, we observed a differentiation in the pattern of activity for posterior regions. There were unique areas of activation in parietal regions for the color-word task and in occipitotemporal regions for the color-object task. No increase in activation was observed in regions responsible for processing the perceptual attribute of color. The results of this second experiment indicate that attentional selection in tasks such as the Stroop task, which contain multiple potential sources of relevant information (e.g., the word vs. its ink color), acts more by modulating the processing of task-irrelevant information than by modulating processing of task-relevant information.

\section{INTRODUCTION}

Researchers investigating the neural bases of attention (LaBerge, 1990) conceive of it as occurring via a network of brain structures that includes the thalamus, pulvinar, superior colliculus, posterior parietal regions, prefrontal regions, anterior cingulate cortex, and basal ganglia (Posner \& Petersen, 1990; Mesulam, 1981). Some researchers have suggested that these structures form two distinct attentional systems, one anterior and one posterior (Posner \& DeHaene, 1994). Traditionally, the anterior system is considered responsible for executive aspects of attention, most notably attention for action. This conceptualization is supported by neuroimaging studies that reveal increased activity in prefrontal and cingulate areas when there is an executive or decisionmaking component to attentional control, such as when attention must be divided across a number of different stimulus attributes (e.g., Corbetta, Miezin, Dobmeyer, Shulman, \& Petersen, 1991). The posterior system, in contrast, is thought to be involved in the selection of information based on perceptual characteristics and/or

spatial location (O'Craven, Rosen, Kwong, Triesman, \& Savoy, 1997; Mangun, 1995; Corbetta et al., 1991). Typically, when individuals direct their attention to one particular attribute of an item (e.g., motion), increased activity is observed in the posterior brain region specialized for processing that visual attribute (e.g., increased activity in the human equivalent of MT or V5: Corbetta et al., 1991).

In this paper we examine a dissociation between functioning of the anterior and posterior subsystems of the attentional network. Here we provide strong evidence for the role of the anterior system in executive aspects of attention, as well as supplying new insights into the organization of prefrontal cortex for such functions. We demonstrate that with increased attentional demands, activation is increased in different subdivisions of prefrontal cortex and precuneus depending upon the nature of the information that is task relevant (e.g., color vs. spatial information). Rather than the prefrontal cortex acting as a unified whole, our data suggest that distinct subregions of the dorsolateral prefrontal cortex 
play a role in imposing executive aspects of attentional control depending upon the nature of the information that is to be selected. As a corollary, we also demonstrate that the pattern of activity within the dorsolateral prefrontal cortex is relatively independent of the type of information that is to be ignored (i.e., task-irrelevant information). This finding would be expected if indeed the dorsolateral prefrontal cortex acts to impose topdown executive control in selecting task-relevant information.

It has also been suggested that prefrontal control over executive aspects of attention feeds backward and tunes posterior cortical systems to enhance the processing of task-relevant information (Cohen, Braver, \& O'Reilly, 1996; Desimone \& Duncan, 1995). It is generally assumed that prefrontal regions "alert" those posterior brain regions responsible for processing the sensory information that is task-relevant. As a result, the sensory areas related to the task-relevant feature become more activated, evidence that processing of the task-relevant sensory feature is being modulated (e.g., lingual fusiform regions will become active when color is being attended compared to form or motion). Here we provide evidence that when task-relevant information is contained in two distinct streams of information rather than only one, there is little modulation of processing of the taskrelevant sensory feature. Rather, we find increased activation in posterior regions that process the task-irrelevant information. These findings are more compatible with the idea that attentional selection occurs via modulation of processing the task-irrelevant information rather than by the more conventional view that the processing of task-relevant information is modulated.

Our evidence for these assertions is based on experiments using variants of the Stroop task. In the classic color-word Stroop task, individuals are asked to identify the ink color in which a word is printed while ignoring its identity. The Stroop task is a particularly useful tool for the investigation of executive aspects of attentional control because it requires more than just attending to one attribute over another. Rather, executive aspects of attentional control are required to suppress the automatic processing of the word's identity while prioritizing the less automatic processing of the word's ink color (MacLeod, 1991, 1992). Consistent with such an analysis of the Stroop task, many theorists posit that one important aspect of executive attentional control is the ability to override routine processes (e.g., Stuss \& Benson, 1986; Shallice, 1982; see also Banich, 1997, Chapter 10 for a review).

Results from the Stroop task demonstrate, however, that the ability to suppress automatic word reading is incomplete. Compared to a neutral baseline word that has no intrinsic meaning with regard to color (e.g., "blank"), responses are speeded if the word names the ink color (e.g., the word "red" in red ink; congruent trials), and responses are slowed if the word names a different ink color (e.g., the word "blue" in red ink; incongruent trials). Generally, responses to incongruent trials are thought to require greater attentional selection than those on neutral trials. On incongruent trials the selection of task-relevant information (i.e., the item's color) must occur in the face of task-irrelevant information that conflicts at the level of meaning (e.g., the meaning of the word "red" conflicts with the ink color blue) and/or at the level of a response (e.g., a response to the word "red" conflicts with selecting the correct response of blue). No such conflict exists for neutral trials.

Prior neuroimaging work indicates that the greater attentional demands imposed by incongruent compared to neutral trials engages a network of neural structures including prefrontal cortex, the anterior cingulate, and posterior regions (e.g., superior parietal cortex, extrastriate cortex) (Brown et al., 1999; Bush et al., 1998; Taylor, Kornblum, Lauber, Minoshima, \& Koeppe, 1997; Carter, Mintun, \& Cohen, 1995; Carter, Mintun, Nichols, \& Cohen, 1997; Bench et al., 1993; Pardo, Pardo, Janer, \& Raichle, 1990). To elucidate the specific role of the anterior and posterior attentional systems in attentional control for the Stroop task, we performed two experiments. In the first experiment, we employed two variants of the Stroop task that differed in the nature of the task-relevant dimension, but shared the same task-irrelevant dimension. By holding the task-irrelevant dimension constant across the two tasks, we could determine whether the increased attentional demands imposed by incongruent compared to neutral trials activated different subregions within a brain area depending on the nature of the task-relevant dimension. In the second experiment, we took the converse approach: We varied the nature of the task-irrelevant dimension across two tasks, but kept the task-relevant dimension constant. By holding the task-relevant dimension constant, we were able to determine whether any brain areas exhibited increased sensitivity to the task-irrelevant dimension on incongruent relative to neutral trials. The findings of these two experiments provide strong converging support indicating that: (1) a dorsolateral prefrontal/precuneus system is involved in executive control for selecting task-relevant information; (2) this system has a modular organization as the specific region responsible for such executive aspects of attentional control varies as a function of the nature of task-relevant information; and (3) when two streams of information (i.e., information contained in the word compared to the ink color) are relevant to task demands, selection occurs more by modulating processing of the task-irrelevant information than by modulating processing of the task-relevant information.

\section{EXPERIMENT 1}

In the first experiment we contrasted the pattern of brain activation for two tasks: a color-word Stroop task 
and a spatial-word Stroop task. We describe our tasks in a hyphenated manner with the first word referring to the task-relevant dimension and the second word referring to the task-irrelevant dimension. As described above, in the color-word Stroop task, color is the relevant dimension whereas the word's identity is the irrelevant dimension. In the spatial-word Stroop task, the spatial relation between a word and a box (i.e., above, below, within) is the relevant dimension whereas the word's identity is the irrelevant dimension.

To manipulate attentional demands in each task, we varied the relation between information in the taskrelevant and task-irrelevant dimensions. Attentional demands were higher on incongruent trials, as the task-irrelevant information directly conflicted with the task-relevant information. An example of an incongruent trial for the color-word task is the word "blue" printed in yellow, and for the spatial-word task, the word "above" positioned below the box. Attentional demands were lower on neutral trials, in which the task-irrelevant information was unrelated to the taskrelevant information. An example of a neutral trial for the color-word task would be the word "blank" colored in yellow, and for the spatial-word task, the word "civil" positioned below the box.

Given that these two Stroop tasks differ in the taskrelevant dimension but not in the task-irrelevant dimension, we predicted that different subregions of the executive control system would be activated as the need for attentional selection was increased. This prediction is based on findings in monkeys (Wilson, O Scalaidhe, \& Goldman-Rakic, 1993) and humans (Smith \& Jonides, 1999) that there is a modularity of organization to the prefrontal cortex that differentiates on the basis of the relevant task domain. In particular, distinct regions of prefrontal cortex are involved in spatial compared to nonspatial working memory. Given the interrelationship between working memory and attentional selection (Desimone \& Duncan, 1995), we expected a similar modularity for executive aspects of attentional selection.

\section{Results}

\section{Behavioral Results}

As expected, responses to incongruent trials were slower compared to neutral trials: significantly so for the color-word task $[679$ msec neutral; 818 msec incongruent; $F(1,12)=46.8, p<.0001]$ and marginally so for the spatial-word task $[637 \mathrm{msec}$ neutral; 668 msec incongruent; $F(1,12)=3.72, p<.10]$. This finding verifies that our attentional manipulation was indeed effective. Furthermore, an analysis revealed a significant interaction $[F(1,12)=16.12, p<.002]$ such that the elongation of response to incongruent trials was significantly greater for the color-word task than the spatial-word task.

\section{Neuroimaging Results}

Figure 1 shows regions that were significantly more active for the incongruent trials compared to neutral trials (mean $Z=1.96, N=14$ ) for each of the two tasks. As in prior Stroop tasks, there was a wide range of areas that exhibited greater activation in incongruent than neutral trials including prefrontal, cingulate, parietal, and occipito-temporal regions. As can be seen in Figure 1, although these regions overlapped for the two tasks, there was differentiation within these general regions based on the nature of the attended attribute.

We performed a secondary analysis (see Methods) to provide confirmation of these dissociations. Our basic approach had two steps. First, we isolated for each task the regions significantly more activated for incongruent
Figure 1. Depicted here are areas of the brain in Experiment 1 that yielded significantly greater activation in the incongruent than neutral condition. Activated regions for the colorword task are shown in orange, those for the spatial-word task are shown in blue, and areas of common activation are shown in purple. (a) Clear differentiation of activation on the basis of the attended attribute can be seen in dorosolateral prefrontal regions with that for the spatial-word Stroop being located more dorsally, and that for the color-word task being located more ventrally. Also note the region of inferior parietal cortex activated only by the spatial-word task. (b) Note a similar differentiation in left inferior parietal cortex with activation located more dorsally for the spatial-word Stroop task than color-word Stroop task.

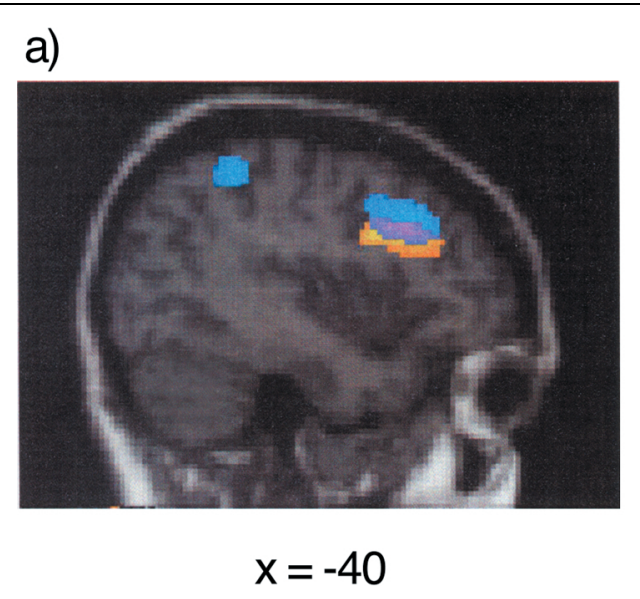

b)

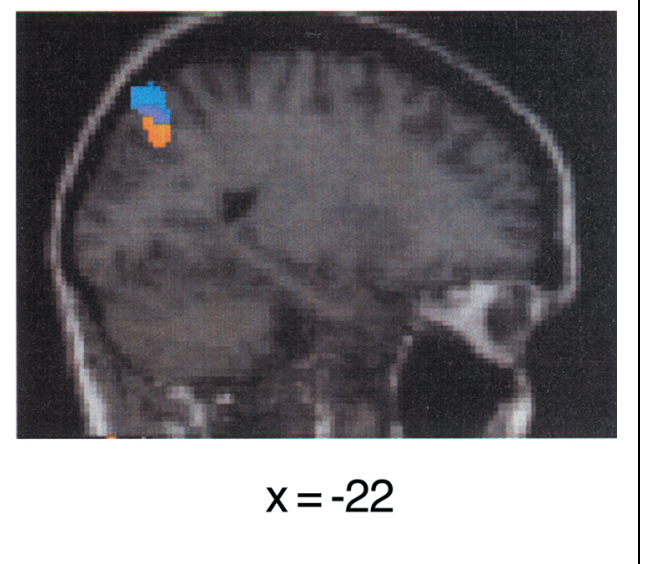


than neutral trials. Second, we then determined which of those regions were significantly more active for one task than the other. The Talairach coordinates for the peaks of these regions are listed in Table 1 . The results of this analysis confirmed our primary analysis, showing a dissociation between the subregions activated by each of the two tasks.

In the prefrontal cortex we found a double dissociation, in that some areas activated by the color-word task were not activated by the spatial-word task, and vice versa. Common regions of significant activation for both tasks occurred in the left middle frontal gyrus (BA 9) (e.g., $x=-42, y=20, z=28$ ). The color-word task, however, produced significantly more activation than the spatial-word task in medial and ventral regions, whereas the spatial-word task produced significantly more activation than the color-word task in dorsal regions (See Figure 1a). In addition, the color-word task produced significantly greater activation in the right middle and inferior frontal regions than did the spatialword task.

A similar, but less robust dissociation was observed for cingulate regions. An area of the right cingulate cortex was significantly more activated by incongruent than neutral trials for the color-word task but not for the spatial-word task. Conversely, there was a region of medial frontal/supplementary motor area (SMA) that was significantly more activated on incongruent than neutral trials for the spatial-word task but not for the color-word task. However, this latter difference in activation between the two tasks did not reach conventional levels of significance in the confirmatory analysis.

A similar dissociation in the patterns of activation for the two tasks was also observed for posterior regions (e.g., parietal and occipito-temporal cortex). Activity was significantly greater for the spatial-word than colorword task in superior regions of parietal cortex, and significantly greater for the color-word than spatial-

Table 1. Peaks of Regions in Experiment 1 Significantly More Activated for One Task Than the Other

\begin{tabular}{|c|c|c|c|c|c|c|c|c|c|}
\hline \multicolumn{5}{|c|}{ Color-word task } & \multicolumn{5}{|c|}{ Spatial-word task } \\
\hline Region & $x$ & $y$ & $z$ & Mean Z & Region & $x$ & $y$ & $z$ & Mean Z \\
\hline \multicolumn{10}{|l|}{ Medial frontal } \\
\hline \multirow[t]{2}{*}{ Anterior cingulate (32) } & 6 & 18 & 40 & 2.70 & & & & & \\
\hline & & & & & Medial frontal (8) & 4 & 26 & 46 & 2.04 \\
\hline \multicolumn{10}{|l|}{ Frontal } \\
\hline Right middle frontal (46) & 40 & 22 & 24 & 2.99 & & & & & \\
\hline \multirow[t]{3}{*}{ Left middle frontal (46) } & -34 & 22 & 24 & 3.23 & & & & & \\
\hline & & & & & Left middle frontal (9) & -42 & 28 & 32 & 2.55 \\
\hline & & & & & Left middle frontal (8) & -40 & 10 & 38 & 3.11 \\
\hline Left inferior frontal (44) & -46 & 6 & 26 & 3.61 & & & & & \\
\hline Right inferior frontal (44) & 44 & 10 & 26 & 3.73 & & & & & \\
\hline \multicolumn{10}{|l|}{ Parietal } \\
\hline \multirow[t]{4}{*}{ Left precuneus (7) } & -22 & -70 & 36 & 4.59 & & & & & \\
\hline & & & & & Left precuneus(7) & -10 & -80 & 54 & 2.39 \\
\hline & & & & & Left inferior parietal (40) & -42 & -42 & 48 & 2.55 \\
\hline & & & & & Right superior parietal (7) & 30 & -72 & 56 & 2.36 \\
\hline \multicolumn{10}{|l|}{ Occipito-temporal } \\
\hline & & & & & Left superior temporal $(22 / 38)$ & -46 & 14 & 0 & 2.86 \\
\hline & & & & & Left middle temporal (39) & -52 & -58 & 18 & 2.97 \\
\hline
\end{tabular}

These peaks are identified by the Brodmann's area within which they fall (noted in parentheses), their Talairach coordinates, and the maximal mean $Z$ score within that peak. Notice that the mean $Z$ value is the average $Z$ value for that region across all subjects. If there was more than one peak of activation within an anatomical/Brodmann's area, only that peak with the highest maximal mean $Z$ is listed. The peaks identified in italics are ones that yielded a significant activation between incongruent and neutral trials in the spatial-word task but not the color-word task, although the difference in activation between the two tasks did not reach significance. 
word task in medial and inferior parietal regions (see Figure 1b). There were, however, common regions in the left inferior parietal cortex (e.g., $x=-22, y=-2, z=$ 46) that were more activated by incongruent than neutral trials for both tasks. In addition, the contrast between incongruent and neutral trials produced a region of significant activation in the left inferior parietal cortex (BA 40) for the spatial-word Stroop task that was not observed for the color-word task (refer back to Figure 1a). It should be noted, however, that the difference in activation between the two tasks did not reach significance in our confirmatory analysis. Finally, there was a region of right superior parietal cortex that revealed greater activation for incongruent than neutral trials for the spatial-word task but not the color-word task.

In addition, the contrast between incongruent and neutral trials for the spatial-word task produced two areas in left temporal regions that were more activated than (and not observed) in the color-word task. One of these was in the very posterior portions of the middle temporal gyrus, and the other in the anterior region of the superior temporal gyrus.

\section{Discussion}

The comparison between incongruent and neutral trials for both Stroop tasks indicated activation of a similar brain network that includes prefrontal, cingulate, and parietal regions. These findings are consistent with other studies involving attentional selection and/or the Stroop task (e.g., Carter et al., 1995; Pardo et al., 1990). Importantly, however, the location of activation within each of these regions varied for each task, indicating a sensitivity of each region to the nature of the taskrelevant dimension. In the case of dorsolateral prefrontal regions, we observed a strong double dissociation between ventral and dorsal regions. Ventral regions showed differential activation with increased attentional demands (i.e., on incongruent compared to neutral trials) in the color-word task, whereas more dorsal regions exhibited differential activation with increased attentional demands in the spatial-word task.

The color-word task yielded significantly greater activation in inferior frontal regions (BA 44) and dorsolateral prefrontal cortex (BA 46) than the spatial-word task. Activity in BA 44 of the left hemisphere is typically associated with phonological processing (e.g., Wagner, 1999; Price, 1998; Fiez, 1997), and in the right hemisphere with visuospatial working memory (e.g., Wagner, 1999). Activation in BA 44 of the left hemisphere could possibly reflect phonological interference between representations of the color word and the ink color, or covert vocalization, as damage in this region typically produces Broca's aphasia.

Activation in BA 46 is typically associated with the manipulation or selection of information that is being held in working memory (Owen, Evans, \& Petrides,
1996; Petrides, 1994). We propose that this dorsolateral prefrontal region becomes activated on incongruent compared to neutral trials in the color-word task because the individual must determine which of the word's attributes (i.e., its meaning or its visual color) contains the relevant color information. Supporting such an interpretation are data from our laboratory (Banich et al., 1999) in which we have found activation in this area for both incongruent and congruent Stroop trials compared to neutral trials. Incongruent and congruent trials are similar in that both require the individual to determine that the relevant color information is contained in the word's ink color compared to its identity.

In contrast, the spatial-word task produced significantly greater activation with increased attentional demands in areas 8 and 9 of the left middle frontal gyrus than did the color-word task. BA 9 is also part of dorsolateral prefrontal cortex, and hence has also been associated with manipulation of information in working memory. The more dorsal activation within dorsolateral prefrontal cortex for the spatial-word task than the color-word task is consistent with findings in animals (Wilson et al., 1993) and humans (Smith \& Jonides, 1999) that regions associated with the processing of spatial information are located more dorsally than those associated with the processing of information related to objects. The activation in BA 8 is near the frontal eye fields in humans (e.g., Petit \& Haxby, 1999). This increased activity on incongruent as compared neutral trials may have occurred because individuals may move their gaze between the box and the word more often to deal with the conflict between the word's meaning and the word's location relative to the box.

A dissociation between the two tasks with increased attentional demands was also observed in regions of the cingulate. A ventral region of the anterior cingulate was significantly more activated in the color-word than spatial-word task. In contrast, the spatial-word task activated a superior region in medial frontal areas that impinges upon the SMA. Once again we observe a more dorsal center of activation in the spatial-word task, consistent with the pattern of anatomical segregation seen for these two types of material in both posterior and frontal regions.

Within parietal regions a double dissociation in activation associated with increased attentional demands was observed in the left precuneus. A more lateral and inferior region was activated by the color-word task, whereas a more medial and superior region was activated by the spatial-word task. This dissociation mimicked that observed for dorsolateral prefrontal regions. Activation in this region has been reported in other attentional tasks, such as when individuals must shift attention between two attributes of an item (e.g., between form and color in a modified Wisconsin Card Sorting Task: Nagahama et al., 1999). Hence, our results are consistent with the suggestion of Goldman-Rakic (1988) that there 
are parallel connections between distinct regions of the prefrontal and parietal cortex. In this case, there appear to be distinct subdivisions within each of these regions that are important for attentional selection involving color compared to space.

The spatial-word task also uniquely activated superior regions of the right parietal lobe and left inferior parietal regions. Both of these regions have been previously described as being part of a general attentional activation system that allocates resources regardless of the stimulus dimension (e.g., Coull \& Nobre, 1998). For example, the lateral left inferior parietal region has been found to be more active in both a space-based and an object-based attention task than in a control task in which length of an object had to be reported (Fink, Dolan, Halligan, Marshall, \& Frith, 1997). Likewise, activation of the right superior parietal region has been found in both a spatial-orienting and temporal-orienting task (Coull \& Nobre, 1998). Our data, however, are somewhat at odds with the idea that such a system is insensitive to the stimulus dimension to be attended, as we did not find activation in these regions for the colorword task. There is another possible interpretation of the increased activation in right superior parietal cortex on incongruent compared to neutral trials. This region has been reported to be involved in stimulus-response (e.g., button press) mapping (e.g., Meyer et al., 1998). In our task, such a mapping might be more difficult on incongruent trials than neutral trials for the spatial-word task but not the color-word task. Whereas task-relevant and task-irrelevant information on incongruent trials in the spatial-word task contain conflicting spatial information that would make a stimuli-response mapping difficult, no such conflict with regard to spatial information occurs in the color-word task.

With regard to the activity in occipito-temporal regions in the spatial-word task, the left middle temporal activation appear to represent processing within early portions of the dorsal visual-processing stream. This region may also help to process relative spatial position as regions just superior to this location in the left hemisphere have been found to be involved in the processing of categorical spatial relations (e.g., above/below; left/right) (Baciu et al., 1999) although others have found the regions involved in categorical spatial relations to be more variable (Kosslyn, Thompson, Gitelman, \& Alpert, 1998). The superior temporal activation may represent the processing of the box and the word as unique objects. Because anterior regions of superior temporal sulcus project to the posterior parietal cortex (Baizer, Ungerleider, \& Desimone, 1991), activation in this region may indicate an integration of the items with respect to their relative positions in space. In summary, we found differentiation of activation depending on the nature of the taskrelevant information in both the anterior and posterior attentional networks.

\section{EXPERIMENT 2}

On the basis of Experiment 1, we concluded that the differential activation within distinct subregions of prefrontal and precuneus cortex with increased attentional demands reflects regional segregation of attentional control for different types of task-relevant information (e.g., spatial vs. color). If that were the case, then one would not predict a double dissociation in regional activation with increased attentional demands if two tasks share the same type of task-relevant information (e.g., color) but differ only with regard to task-irrelevant information. To test this hypothesis, our second study examined patterns of activation for a color-word and a color-object Stroop task. In the color-object Stroop task, an individual must name the color in which an object is presented, while disregarding the identity of the object. Because these tasks only differ in the task-irrelevant dimension, any brain region that shows a difference in activity between the two tasks must be sensitive to the taskirrelevant information.

Once again, we varied attentional demands by comparing incongruent trials, which require greater attentional selection because the task-irrelevant information conflicts with the task-relevant information, to neutral trials, which require less attentional selection because they contain no such conflict. Incongruent trials in the color-object Stroop task are those in which the item is depicted in a color different than the one with which it is

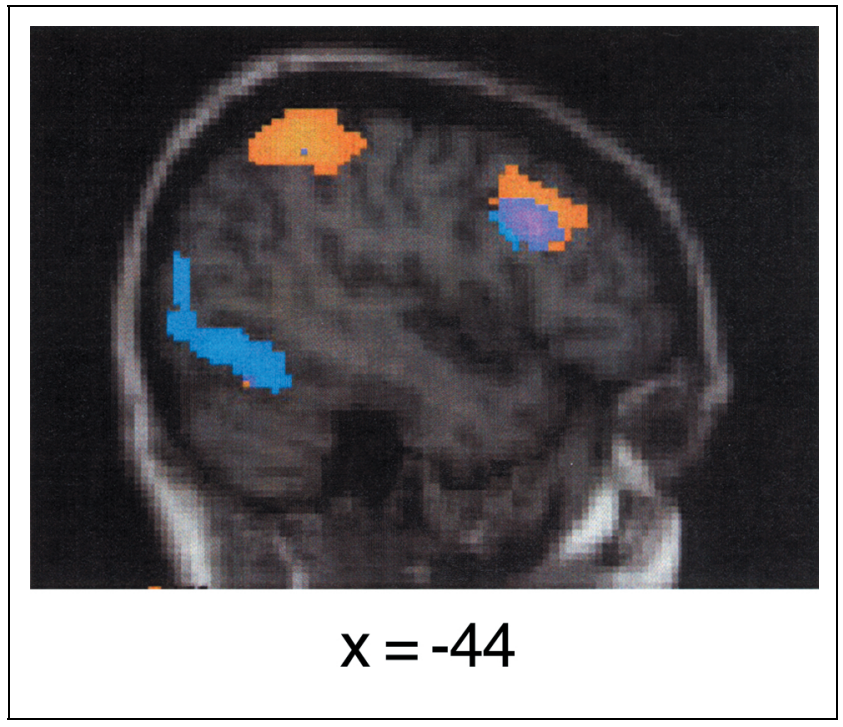

Figure 2. Depicted here are areas of brain activation that were significantly greater in the incongruent than neutral condition in the second experiment. Activated regions for the color-word task are shown in orange, those for the color-object task are shown in blue, and areas of common activation are shown in purple. Highly overlapping areas of activation for the two tasks were observed in the prefrontal cortex, whereas the color-word task activated parietal cortex and the color-object task activated the temporo-parietal cortex. 
strongly associated (e.g., a yellow strawberry when strawberries are strongly associated with the color red). Neutral trials are those in which the object is not strongly associated with any particular color (e.g., a yellow car).

\section{Results}

\section{Behavioral Results}

Responses were significantly slower on incongruent trials compared to neutral trials [color-word task: neutral $577 \mathrm{msec}$, incongruent $692 \mathrm{msec}, F(1,15)=17.92$, $p<.001$; color-object task: neutral $569 \mathrm{msec}$, incongruent 657 msec, $F(1,15)=7.12, p<.025]$. These behavioral data indicate, once again, that our manipulation of attentional demand was effective. However, the increase in reaction time on incongruent compared to neutral trials was no greater for the color-word task than for the color-object task.

As shown in Figure 2, the two tasks activated similar regions of the anterior executive attentional system, but showed greater differences in regions of activation of the posterior attentional system. As in Experiment 1, we performed a confirmatory analysis to verify these findings.

Highly overlapping regions of frontal cortex were activated by the two tasks including left and right middle frontal gyrus (BA 9 and BA 46). Unlike Experiment 1, in which unique prefrontal regions were activated for each task, such a pattern of double dissociation was not observed here (see Figure 2). Rather, three regions exhibited greater activation with increased attentional demands in the color-word task that were not observed

Table 2. Peaks of Regions in Experiment 2 Significantly More Activated for One Task Than the Other

\begin{tabular}{|c|c|c|c|c|c|c|c|c|c|}
\hline \multicolumn{5}{|c|}{ Color-word task } & \multicolumn{5}{|c|}{ Color-object task } \\
\hline Region & $x$ & $y$ & $z$ & Mean Z & Region & $x$ & $y$ & $z$ & Mean $Z$ \\
\hline \multicolumn{10}{|l|}{ Medial frontal } \\
\hline $\mathrm{R}$ medial frontal $(8 / 6)$ & 4 & 16 & 48 & 3.27 & & & & & \\
\hline \multicolumn{10}{|l|}{$\underline{\text { Frontal }}$} \\
\hline Left middle frontal (8) & -38 & 12 & 40 & 2.37 & & & & & \\
\hline Right middle frontal (6) & 34 & 10 & 50 & 2.68 & & & & & \\
\hline \multicolumn{10}{|l|}{ Parietal } \\
\hline Left precuneus (7) & -6 & -62 & 50 & 2.76 & & & & & \\
\hline Right precuneus (7) & 2 & -60 & 62 & 2.63 & & & & & \\
\hline Left superior parietal (7) & -26 & -60 & 62 & 2.77 & & & & & \\
\hline Left inferior parietal (40) & -40 & -52 & 48 & 3.31 & & & & & \\
\hline \multicolumn{10}{|l|}{ Occipito-temporal } \\
\hline & & & & & Left fusiform (37) & -40 & -54 & -8 & 2.78 \\
\hline & & & & & Left inferior occipital (19) & -38 & -70 & -2 & 2.78 \\
\hline & & & & & L inferior occipital (18) & -42 & -80 & 0 & 2.78 \\
\hline & & & & & Left lingual (18) & -16 & -88 & 2 & 3.8 \\
\hline & & & & & Right middle occipital (18) & 20 & -86 & 12 & 4.59 \\
\hline & & & & & Left middle occipital (18) & -24 & -84 & 12 & 4.86 \\
\hline & & & & & Left middle temporal (19/39) & -26 & -76 & 22 & 3.95 \\
\hline & & & & & Right middle temporal $(19 / 39)$ & 36 & -72 & 26 & 3.32 \\
\hline & & & & & Left precuneus $(7 / 31)$ & -16 & -64 & 40 & 2.44 \\
\hline
\end{tabular}

These peaks are identified by the Brodmann's area within which they fall (noted in parentheses), their Talairach coordinates, and the maximal mean $Z$ score within that peak. Notice that the mean $Z$ value is the average $Z$ value for that region across all subjects. If there was more than one peak of activation within an anatomical/Brodmann's area, only that peak with the highest maximal mean $Z$ is provided. 
for the color-object task: (1) medial frontal cortex, (2) left middle frontal cortex, and (3) right middle frontal cortex. In all cases, these activations appear to reflect the recruitment of additional areas in the color-word compared to the color-object task rather than a dissociation between regions of activation for the two tasks (See Table 2).

In contrast, regions in parietal and occipito-temporal cortex activated by increased attentional demands were more clearly differentiated on the basis of the taskirrelevant information (see Figure 2). Although both tasks produced bilateral activity in the parietal lobes (BA 7 and BA 40), the patterns of activity significantly differed in that (1) the precuneus cortex was significantly active for the color-word task but not the colorobject task, (2) a superior region of the left superior parietal region was active for the color-word task but not the color-object task, and (3) a lateral region of left inferior regions was more active for the color-word than color-object task. Therefore, although there was parietal activation for both tasks, there were parietal regions uniquely activated by the color-word task.

For the occipito-temporal regions, the only common activation was in the left fusiform gyrus (BA 19). Areas significantly more activated for the color-object task than the color-word task extended throughout the ventral visual processing stream, including the inferior occipital, middle occipital, fusiform, lingual, and middle temporal gyri. This pattern was more pronounced in the left hemisphere than the right.

It is of note that we specifically examined whether activation in the color-processing region (e.g., $x=-12$, $y=-74, z=0$ ) was modulated with increased attentional demands. No such increase in activation in this region was observed for either task.

\section{Discussion}

In this experiment, unlike Experiment 1, we found clear evidence of a dissociation in processing between the anterior and posterior attentional systems. The posterior system was sensitive to task-irrelevant information whereas the anterior system was much less so. Our finding that the two tasks activated highly overlapping regions of prefrontal cortex is consistent with the suggestion that prefrontal regions are acting in an executive manner to select the task-relevant information. We, like others (Paus, Petrides, Evans, \& Meyer, 1993), interpret the increased activity in cingulate areas to reflect a mechanism for attention for action, in this case for response selection.

Whereas some posterior regions exhibited increased activation with increased attentional demands in both tasks, the most striking feature of our data was the dissociation between the two tasks in the pattern of activation in parietal and occipito-temporal regions. Although there were common regions of increased activation for the two tasks in the parietal lobes, a number of parietal regions were more activated by the color-word than color-object task. These regions appear to be related to word processing. For example, the region of the left precuneus that exhibited increased activation in our study has been reported to become active when processing words compared to letter strings (Jessen et al., 1999). Notice that this region of the precuneus is different than that which showed differential activation in the color-word and spatial-word tasks in Experiment 1. The significantly greater activation of the lateral left inferior region in the color-word than color-object task is likely to reflect the translation of orthography to phonology (e.g., Price, 1998), as this area has been reported to be more active when viewing or naming words compared to pictures. Finally, the left superior region becomes activated when words must be encoded into memory (Kelley et al., 1998).

With regard to occipito-temporal regions, we found that a significant increase in activity was noted for both tasks in the left fusiform gyrus. What was much more striking, however, was that most all of the ventral visual processing stream was more activated by the colorobject than color-word task. These regions are strikingly similar to those activated when objects are encoded into memory (Kelley et al., 1998). It is important to note that activity in this ventral visual processing stream did not include the area in the left lingual gyrus that has been previously identified as being the area associated with color perception, nor areas in the inferior temporal lobe (BA 20) that are associated with retrieving color knowledge (Chao \& Martin, 1999). Such a pattern of results supports our assertion that activation of posterior regions is being modulated by task-irrelevant compared to task-relevant information.

One might question why task-irrelevant rather than task-relevant information is modulating activity of posterior regions. In the case of incongruent trials in the Stroop task, both the task-relevant and task-irrelevant attributes contain information associated with color (e.g., the word "green" is associated with a color, as is a frog). Prior studies have suggested an increased gain in posterior regions that process an attended attribute such as color. However, we did not observe any additional increase in processing in the color region when we increased the need to attend to the color in the face of conflicting information (e.g., a color-related word, a color-related object). Rather, we observed increased activation for those regions of the brain associated with processing the task-irrelevant information. Hence, it appears that when there is conflicting information, attentional control occurs more by modulating processing of the task-irrelevant information than by modulating processing of task-relevant information.

We speculate that such increased activation in regions responsible for processing task-irrelevant information may reflect the detection that this information is related 
to the information that is task-relevant, rather than that it conflicts with task-relevant information. This speculation is based on findings that we have found these same regions to become activated on congruent compared to incongruent trials (Banich et al., 1999). Detecting that the task-irrelevant information is related to task-relevant information appears to occur early on in processing. The regions of increased activation observed in the colorword and color-object tasks are not those that reflect the final endpoint of processing for such types of information in the brain. For the color-word task, the increased activation was observed in those regions that translate orthography to phonology, rather than in insula areas involved in semantic processing. It may be that the brain attempts to hinder the processing of taskirrelevant information in the word channel before it reaches semantic processing areas as the semantic representation of the word would more potently interfere with task demands. Similarly, the increased activation in the color-object task was observed in early portion the ventral visual processing stream-in regions in which color and objects are more intimately bound, rather than in more anterior areas that are associated with the detailed recognition of specific complex forms. Once again, detection of the relationship between task-irrelevant and task-relevant information at this level may help to preclude further processing of the task-irrelevant information that would only make it a more potent source of interference.

\section{GENERAL DISCUSSION}

Across the two experiments, we have clearly demonstrated a differentiation in the types of information that influence the selection processes performed by the anterior and posterior attentional subsystems. Whereas both systems are sensitive to task-relevant information, the posterior system is also largely influenced by taskirrelevant information.

Here we consider the processes by which each of these systems is involved in attentional selection. We propose that the dorsolateral prefrontal/precuneus system is responsible for executive aspects of attentional selection. In particular, we suggest that this system imposes an attentional "set" for the task-relevant information-that is, sets a top-down bias for selecting certain types of information (e.g., color). Single-cell recording in animals (e.g., Rainer, Asaad, \& Miller, 1998), neuropsychological studies (e.g., Lombardi et al., 1999; Vendrell et al., 1995), and computational modeling (e.g., Cohen et al., 1996) are consistent with such a suggestion, as all implicate prefrontal regions in acting to impose top-down control over selection of an attended attribute. The results of the present study and other recent work in our laboratory suggest that these dorsolateral prefrontal areas are especially invoked in this process when selection is attentionally difficult.
Consider that reading a word to retrieve its meaning is relatively automatic (e.g., Cohen, Dunbar, \& McClelland, 1990), whereas extracting color information from a word or noting its spatial location are much less so. We propose that dorosolateral prefrontal regions are required to direct attention to the task-relevant information, such as a word's color or its spatial location, when these types of information are typically ignored. To provide support for this idea we performed another study (Banich et al., 2000) in which we examined the degree of dorosolateral prefrontal activity under two conditions. In one condition, it was easier to direct attention to the task-relevant dimension of a stimulus compared to the task-irrelevant dimension. In the other, it was more difficult to direct attention to the taskrelevant dimension compared to the task-irrelevant dimension. We only obtained significant dorsolateral prefrontal activity in the latter case, consistent with the idea that dorsolateral prefrontal regions are only involved when one needs executive control to direct attention to the task-relevant dimension, and not when such information is processed automatically.

Although the pattern of activation for cingulate cortex was similar to that of dorsolateral prefrontal cortex in that distinct subdivisions were activated by the colorword compared to spatial-word task, we have numerous reasons to believe that the cingulate's role in attentional selection is distinct from that of dorsolateral prefrontal cortex. Although a detailed discussion of these reasons is beyond the scope of this paper, we will provide a brief synopsis here. In the study discussed above in which we varied the relative difficulty of attending to the taskrelevant compared to task-irrelevant dimension, we found no relation of this manipulation to activity in the cingulate (Banich et al., 2000). Furthermore, in another study we varied the degree of response interference engendered by different variants of the Stroop task. The results of this investigation suggested that the increases in the cingulate's activity are primarily driven by the degree to which task-irrelevant information provides information about possible responses (Milham et al., 1999).

In contrast to activity in anterior regions, activity in posterior regions is influenced by task-irrelevant information. This finding is interesting as it suggests that selection may occur by a modulation of processing of task-irrelevant information rather than by enhanced processing of task-relevant information. In many other studies of attentional selection, researchers have noted an increased activation in the brain region responsible for processing perceptual information about the attended attribute or feature (e.g., Corbetta et al., 1991, Wojciulik, Kanwisher, \& Driver, 1998). In their studies, however, unlike ours, task-irrelevant information is not related to task-relevant information. For example, in those studies the information provided by an item's form or its motion does not provide information about 
its color. In contrast, on incongruent trials in the Stroop task, the task-irrelevant information conflicts with the task-relevant information. From this perspective it should not be surprising to find an effect of taskirrelevant information in Stroop tasks, as interference is posited to occur on incongruent trials specifically because processing of the task-irrelevant information cannot be totally inhibited.

The fact that activity in these posterior regions increased on incongruent compared to neutral trials might seem counterintuitive, as the incongruent trials require greater selection against the word and object information, respectively, than do neutral trials. Therefore, one might have expected a decrease, rather than an increase, in activation in these regions. However, activation in these regions may increase because the task-irrelevant information on incongruent trials is associated with that which has been deemed as task-relevant (e.g., color), whereas the task-irrelevant information on neutral trials is not.

We speculate that the imposition of an attentional set for task-relevant information by prefrontal regions involves "alerting" all brain regions that process information related to that attentional set. For example, when color is to be attended, other brain regions associated with color processing will be primed to become activated as well. These regions would include those that process the semantic or linguistic value of color or object-processing regions because color is a very salient cue for distinguishing among objects (e.g., Plaut \& Farah, 1990). Activity in these posterior regions would then increase as the brain attempts to resolve the mismatch between the bias set by prefrontal areas for the task-relevant dimension and the presence of the related but incongruent task-irrelevant information in the posterior regions. This attempted resolution would likely occur via a reverberatory loop between frontal and posterior regions (Goldman-Rakic, 1988).

We have considered the possibility that activation of posterior regions is not driven by processing of the taskirrelevant attribute, but rather is linked to some other factor, such as the novelty of seeing an item in an unexpected color (e.g., a yellow strawberry). However, a number of considerations mitigate against this possibility. First, it is traditionally prefrontal areas, rather than posterior areas, that appear to be most sensitive to novelty (Knight, 1984). Second, the results of an unpublished study (Banich et al., unpublished data) revealed that most of the same extrastriate areas showing increased activation in our current study to incongruent color-object stimuli (e.g., a yellow strawberry) relative to neutral stimuli, also yield increased activity to congruent color-object stimuli (e.g., a red strawberry) relative to neutral stimuli. Such a finding precludes the possibility that the novelty of seeing an item in an unexpected color drives activation in these regions. Third, the regions that become activated in our study are distinct from those that show greater activity for items presented in an abnormal color compared to their normal one (Zeki \& Marini, 1998). Hence, we believe that the posterior regions activated in our study are involved in processes associated with attentional selection rather than color perception.

We have also considered and rejected the possibility that the differences in patterns of activation can be accounted for by differences in the overall attentional difficulty imposed by the different variants of the Stroop task. Very distinct regions of posterior cortex showed increased activation in the color-word and color-object Stroop task (Experiment 2), even though the increase in RT caused by incongruent compared to neutral trials did not differ significantly between these two tasks. Potentially more worrisome, however, was the differentiation in activation for the spatial-word and color-word tasks (Experiment 1), the former of which yielded less interference in the behavioral measure on incongruent trials than did the latter. Although there has been some discussion that dorsolateral regions may become activated by more demanding tasks whereas inferior frontal regions may become activated by less demanding tasks, we know of no study suggesting a dissociation within dorsolateral prefrontal cortex, which is what we observed. Furthermore, the nature of the dissociation, with a more dorsal center of activation for the spatial-word task and a more ventral one for the color-word task is compatible with a dissociation between a task-relevant attribute being processed by subdivisions of the visual processing stream, dorsal versus ventral, respectively. Finally, we (Banich et al., 1999) have found that the same region of dorsolateral prefrontal cortex that we observed in both studies to become activated by incongruent compared to neutral color-word trials is also differentially activated by congruent trials (the word "red" in red) compared to neutral trials. If the differentiation in dorsolateral prefrontal cortex were being driven by the difficulty of selecting the ink color, then we would have anticipated that the location of activation in the congruent condition should have been closer to that observed in our spatial-word task. We would have anticipated such a result because the behavioral data suggest that the spatial-word task is less attentionally demanding than the color-word task.

In light of our findings from the first experiment, the results from the second experiment suggest that activation in posterior regions can be modulated by both taskrelevant and task-irrelevant information. In contrast, a double dissociation in the pattern of prefrontal activation was only observed in the first study where the nature of task-relevant information varied, but not in the second study in which the nature of such information was held constant.

Taken together, the results of these studies suggest two distinct mechanisms for modulation of attentional selection. One mechanism, involving prefrontal regions 
and associated areas of the precuneus, is likely to reflect a top-down executive mechanism for imposing an attentional set for task-relevant information. Of most interest, this subsystem appears to have a modular organization with distinct regions of control based on the nature of the task-relevant information (e.g., information in the dorsal vs. ventral visual processing stream). To our knowledge, we are the first to demonstrate such an effect. It should be noted that this finding is compatible with evidence for modularity within the prefrontal cortex for the control of other cognitive functions. For example, recent work suggests the possibility of distinct anatomical localization for spatial compared to nonspatial working memory in humans (Smith \& Jonides, 1999).

The second subsystem, centered in posterior regions, appears to reflect a mechanism whereby the relationship between task-relevant and task-irrelevant information is detected. It appears that when task-irrelevant information is related to the attentional set imposed by frontal regions, processing is modulated not by turning up the gain on posterior regions that process the task-relevant attribute, but rather by a modulation of processing of the task-irrelevant information.

\section{METHODS}

Fourteen volunteers participated in Experiment 1, and 16 volunteers participated in Experiment 2. All were right-handed, between the ages of 20 and 35, had no known history of neurological impairment, and gave informed consent before participating in the study.

In both experiments, each individual participated in four runs. In Experiment 1, half of these runs involved the color-word task and half involved the spatial-word task. In Experiment 2, half of these runs involved the color-word task and half involved the color-object task. Task order was counterbalanced across participants. For each task, the first run consisted of four and a half cycles that were composed entirely of neutral trials. This run familiarized subjects with the task as well as provided evidence for the ability of our statistical criteria to control for false positives in our analyses (see below). The second run consisted of four and half cycles in which a block of neutral stimuli (off phase) was alternated with a block of stimuli that contained half-neutral and half-incongruent trials (on phase). Each half cycle contained 18 trials. A trial appeared once every $2 \mathrm{sec}$. Each trial consisted of a fixation cross for $300 \mathrm{msec}$, followed by the Stroop stimuli for $1200 \mathrm{msec}$. This was followed by a 500-msec intertrial interval. Reaction time was recorded from stimulus onset. Trials in which participants did not respond within $1200 \mathrm{msec}$ were counted as errors. It should be noted that error rates were so low as to make analysis of accuracy irrelevant. The first run for each task (which consisted entirely of the neutral trials) provided adequate practice to provide ceiling levels of performance on accuracy.
For the color-word task, participants pressed one of three buttons to indicate whether the word's ink color was blue, yellow, or green regardless of the meaning of the word. For the spatial-word tasks, participants pressed one of three buttons to indicate whether the word was positioned above, below, or inside the box, regardless of the meaning of the word. For the colorobject task, they pressed one of three buttons to indicate whether the object was green, yellow, or orange, regardless of the color with which it is strongly associated.

Multislice images of the brain were acquired using a 1.5-T GE Signa scanner equipped for echo-planar imaging (EPI). For each run, a total of 144 EPI images were acquired $(\mathrm{TR}=2400 \mathrm{msec}$, $\mathrm{TE}=40 \mathrm{msec}$, flip angle for Experiment $1=60^{\circ}$, flip for angle for Experiment $2=$ $90^{\circ}$ ), each consisting of 11 contiguous slices in Experiment 1 and 15 slices in Experiment 2 (thickness $=7 \mathrm{~mm}$, in-plane resolution $=3.75 \mathrm{~mm}$ ). In addition, a $3-\mathrm{D}$ gradient echo anatomical set was acquired for each participant for the purpose of landmark selection. The head coil was fitted with a bite bar to minimize motion during the sessions.

The first six volumes of each run were discarded to allow the MR signal to reach steady state. Prior to statistical tests, images in the data series were intensity normalized, convolved with a 3-D Gaussian kernel $(\mathrm{FWHM}=8 \mathrm{~mm} \times 8 \mathrm{~mm} \times 8 \mathrm{~mm}$, kernel width $=5$ $\times 5$ ), temporally denoised using an ID-wavelet transform (visu-shrink, number of levels $=4$ ) and linearly detrended (parameter estimates based upon images in the off phases only).

\section{Analysis to Detect Regions Exhibiting Greater Activity on Incongruent Than Neutral Trials}

The Kolmorgorov-Smirnov statistical test was used to generate statistical probability maps between the on and off phases for each voxel for each run. A 4.8-sec hemodynamic lag was assumed. The probability values from the Kolmorgorov-Smirnov test were then converted to $Z$ scores and averaging of $Z$ score maps across subjects was carried out in a common stereotaxic space (Talairach \& Tournoux, 1988). MedX V3.2 was used to carry out image processing and statistical analyses. Using the mean $Z$ score maps, activation maps were generated with a statistical threshold of mean $Z>1.96$. To verify the validity of this threshold, we utilized the runs in which the on and off conditions consisted of only neutral trials. If our threshold were valid, the on/off comparisons under such conditions should not yield any activation, which was indeed the case.

\section{Confirmatory Analysis for Dissociation of Activity Between Tasks}

First we isolated those voxels for which significant increases in activity were noted in our comparison of 
incongruent versus neutral for one task, but not the other (e.g., activity noted in the spatial-word task but not the color-word task, or vice versa). Next, for each of these voxels, we calculated the percent change in the mean BOLD signal (incongruent-neutral) measured from each subject for each task. Finally, for each voxel, we entered these measures into a paired $t$ test, comparing the percent changes observed for colorword and spatial-word tasks, using a significance threshold of $Z>2.5$. Peaks were identified using the Mintun peak detection algorithm (Mintun, Fox, \& Raichle, 1989).

\section{Acknowledgments}

This research was funded by the Beckman Institute for Advanced Science and Technology and performed with support from Carle Clinic. We also thank two anonymous reviewers, Mark D'Esposito, who served as the action editor, and Ben Jacobson for helpful comments.

Correspondence and requests for reprints should be sent to Marie Banich, PhD, Department of Psychology, University of Colorado, Boulder, CO. 80309 (mbanich@psych.colorado.edu).

\section{REFERENCES}

Bacui, M., Koenig, O., Vernier, M.-P., Bedoin, N., Rubin, C., \& Segebarth, C. (1999). Categorical and coordinate spatial relations: fMRI evidence for hemispheric specialization. NeuroReport, 10, 1373-1378.

Baizer, J. S., Ungerleider, L. G., \& Desimone, R. (1991). Organization of visual inputs to the inferior temporal and posterior parietal cortex in macaques. Journal of Neuroscience, 11, 168-190.

Banich, M., Milham, M., Webb, A., Wszalek, T., Brown, C., Gullet, D., Shah, C., Barad, V., Cohen, N., Kramer, A., \& Liang, Z.-P. (1999). Components of the neural network underlying the Stroop task as assessed by fMRI. Society for Neuroscience Abstracts, 25, 865.3.

Banich, M. T. (1997). Neuropsychology: The neural bases of mental function. Boston: Houghton-Mifflin.

Banich, M. T., Milham, M. P., Atchley, R. A., Cohen, N. J., Webb, A., Wszalek, T., Kramer, A. F., Liang, Z.-P., Barad, V., Gullett, D., Shah, C., \& Brown, C. (2000). The unique role of prefrontal regions for imposing an attentional "set": Evidence from fMRI. Cognitive Brain Research, 10, 1-9.

Bench, C. J., Frith, C. D., Grasby, P. M., Friston, K. J., Paulesu, E., Frackowiak, R. S. J., \& Dolan, R. J. (1993). Investigations of the functional anatomy of attention using the Stroop test. Neuropsychologia, 31, 907-922.

Brown, G. G., Kinderman, S. S., Siegle, G. J., Granholm, E., Wong, E. C., \& Buxton, R. B. (1999). Brain activation and pupil response during covert performance of the Stroop Color Word task. Journal of the International Neuropsychological Society, 5, 308-319.

Bush, G., Whalen, P. J., Rosen, B. R., Jenike, M. A., McInerney, S. C., \& Rauch, S. L. (1998). The counting Stroop: An interference task specialized for functional neuroimagingvalidation study with functional MRI. Human Brain Mapping, 6, 270-282.

Carter, C., Mintun, M., \& Cohen, J. D. (1995). Interference and facilitation effects during selective attention: $\mathrm{An}_{2}{ }^{15} \mathrm{O}$ PET study of Stroop task performance. Neuroimage, 2, 264-272.
Carter, C. S., Mintun, M., Nichols, T., \& Cohen, J. D. (1997). Anterior cingulate gyrus dysfunction and selection attention deficits in schizophrenia: $\left[{ }^{15} \mathrm{O}\right] \mathrm{H}_{2} \mathrm{O}$ PET study during single-trial Stroop task performance. American Journal of Psychiatry, 154, 1670-1675.

Chao, L. L., \& Martin, A. (1999). Cortical regions associated with perceiving, naming and knowing about colors. Journal of Cognitive Neuroscience, 11, 25-35.

Cohen, J. D., Braver, T. S., \& O'Reilly, R. C. (1996). A computational approach to prefrontal cortex, cognitive control and schizophrenia: Recent developments and current challenges. Philosophical Transactions of the Royal Society of London, Series B: Biological Sciences, 351, 15151527.

Cohen, J. D., Dunbar, K., \& McClelland, J. L. (1990). On the control of automatic processes: A parallel distributed processing account of the Stroop effect. Psychological Review, 97, 332-361.

Corbetta, M., Miezin, F. M., Dobmeyer, S., Shulman, G. L., \& Petersen, S. E. (1991). Selective and divided attention during visual discrimination of color, shape and speed: Functional anatomy by positron emission tomography. Journal of Neuroscience, 11, 2383-2402.

Coull, J. T., \& Nobre, A. C. (1998). Where and when to pay attention: the neural systems for directing attention tospatial locations and to time intervals as revealed by both PET and fMRI. Journal of Neuroscience, 18, 74267435.

Desimone, R., \& Duncan, J. (1995). Neural mechanisms of selective visual attention. Annual Review of Neuroscience, 19, 193-222.

Fiez, J. A. (1997). Phonology, semantics, the role of the left inferior prefrontal cortex. Human Brain Mapping, 5, 79-83.

Fink, G. R., Dolan, R. J., Halligan, P. W., Marshall, J. C., \& Frith, C. D. (1997). Space-based and object-based visual attention: shared and specific neural domains. Brain, 120, 2013-2028.

Goldman-Rakic, P. (1988). Topography of cognition: parallel distributed networks in primate association cortex. Annual Review of Neuroscience, 11, 137-156.

Jessen, F., Erb, M., Klose, U., Lotze, M., Grodd, W., \& Heun, R. (1999). Activation of human language processing brain regions after the presentation of random letter strings demonstrated within event-related functional magnetic resonance imaging. Neuroscience Letters, 270, 13-16.

Kelley, W. M., Miezin, F. M., McDermott, K. B., Buckner, R. L., Raichle, M. E., Cohen, N. J., Ollinger, J. M., Akbudak, E., Conturo, T. E., Synder, A. Z., \& Petersen, S. E. (1998). Hemispheric specialization in human dorsal cortex and medial temporal lobe for verbal and nonverbal memory encoding. Neuron, 20, 927-936.

Knight, R. T. (1984). Decreased response to novel stimuli after prefrontal lesions in man. Electroencephalography and Clinical Neurophysiology: Evoked Potentials, 59, 9-20.

Kosslyn, S. M., Thompson, W. L., Gitelman, D. R., \& Alpert, N. M. (1998). Neural systems that enode categorical versus coordinate spatial relations: PET investigations. Psychobiology, 26, 333-347.

LaBerge, D. L. (1990). Attention. Psychological Science, 1, 156161.

Lombardi, W. J., Andreason, P. J., Sirocco, K. Y., Rio, D. E., Gross, R. E., Umhau, J. C., \& Hommer, D. W. (1999). Wisconsin Card Sorting Test performance following head injury: Dorsolateral fronto-striatal circuit activity predicts perseveration. Journal of Clinical and Experimental Neuropsychology, 21, 2-16.

MacLeod, C. M. (1991). Half a century of research on the Stroop effect: An integrative review. Psychological Bulletin, 109, 163-203. 
MacLeod, C. M. (1992). The Stroop task: The "gold standard" of attentional measures. Journal of Experimental Psychology, General, 121, 12-14.

Mangun, G. R. (1995). Neural mechanisms of visual selective attention. Psychophysiology, 32, 4-18.

Mesulam, M. -M. (1981). A cortical network for directed attention and unilateral neglect. Annals of Neurology, 10, 309-325.

Meyer, D. E., Evans, J. E., Lauber, E. J., Bmeindl, L., Rubinstein, J., Junck, L., \& Koeppe, R. A. (1998). The role of dorsolateral prefrontal cortex for executive cognitive processes in task switching. Poster presented at the annual meeting of the Cognitive Neuroscience Society, San Francisco, CA.

Milham, M., Banich, M., Webb, A., Barad, V., Cohen, N. J., Wszalek, T., Kramer, A., Liang, Z.-P., Gullett, D., Shah, C., \& Brown, C. (1999). Activity of cingulate based attentional system in Stroop task is dependent upon response eligibility: A hybrid blocked/event-related fMRI design. Neuroimage, 6 , S751.

Mintun, M., Fox, P., \& Raichle, M. (1989). Highly accurate method of localizing regions of neuronal activation in the human brain with positron emission tomography. Journal of Cerebral Blood Flow and Metabolism, 1, 96-103.

Nagahama, Y., Okada, T., Katsumi., Y. Hayashi, T., Yamauchi, H., Sawamto, N., Toma, K., Nakamura, K., Hanakawa, T., Konishi, J., Fukuyama, H., \& Shibasaki, H. (1999). Transient neural activity in the medial superior frontal gyrus and precuneus time locked with attention shift between object features. Neuroimage, 10, 193-199.

O'Craven, K. M., Rosen, B. R., Kwong, K. K., Triesman, A., \& Savoy, R. L. (1997). Voluntary attention modulates fMRI activity in human MT/MST. Neuron, 18, 591-598.

Owen, A. A., Evans, A. C., \& Petrides, M. (1996). Evidence for a two-stage model of spatial working memory processing within the lateral frontal cortex: a positron emission tomography study. Cerebral Cortex, 6, 31-38.

Pardo, J. V., Pardo, P. J., Janer, K. W., \& Raichle, M. E. (1990). The anterior cingulate cortex mediates processing selection in the Stroop attentional conflict paradigm. Proceedings of the National Academy of Sciences, U.S.A., 87, 256-259.

Paus, T., Petrides, M., Evans, C., \& Meyer E. (1993). Role of the human anterior cingulate cortex in the control of oculomotor, manual, and speech responses: a positron emission tomography study, Journal of Neurophysiology, 70, 453-469.
Petit, L., \& Haxby, J. V. (1999). Functional anatomy of pursuit eye movements in humans as revealed by fMRI. Journal of Neurophysiology, 81, 463-471.

Petrides, M. (1994). Frontal lobes and behavior. Current Opinions in Neurobiology, 4, 207-211.

Plaut, D. C., \& Farah, M. J. (1990). Visual object representation: interpreting neurophysiological data within a computational framework. Journal of Cognitive Neuroscience, 2, 320-343.

Posner, M. I., \& DeHaene, S. (1994). Attentional networks. Trends in Neurosciences, 17, 75-79.

Posner, M. I., \& Petersen, S. E. (1990). The attention system of the human brain. Annual Review of Neuroscience, 13, 2542.

Price, C. J. (1998). The functional anatomy of word comprehension and production. Trends in Cognitive Sciences, 2, 281-288.

Rainer, E., Asaad, W. F., \& Miller, E. K. (1998). Selective representation of relevant information by neurons in the primate prefrontal cortex. Nature, 393, 577-579.

Shallice, T. (1982). Specific impairments of planning. Philosophical Transactions of the Royal Society of London, B: Biological Sciences, 298, 199-209.

Smith, E. E., \& Jonides, J. (1999) Storage and executive processes in the frontal lobes. Science, 283, 1657-1661.

Stuss, D. T., \& Benson, F. (1986). The frontal lobes. New York: Raven.

Talairach, J., \& Tournoux, P. (1988). Co-planar stereotactic atlas of the buman brain, 2nd ed. Stuttgart: Thieme.

Taylor, S. F., Kornblum, S., Lauber, E. J., Minoshima, S., \& Koeppe, R. A. (1997). Isolation of specific interference processing in the Stroop task: PET activation studies. Neuroimage, 6, 81-92.

Vedrell, P. Junque, C., Pujol, J., Jurado, M. A., Molet, J., \& Grafman, J. (1995). The role of prefrontal region in the Stroop task. Neuropsychologia, 33, 341-352.

Wagner, A. D. (1999). Working memory contributions to human learning and remembering. Neuron, 22, 19-22.

Wilson, F. A. W., O Scalaidhe, S. P., \& Goldman-Rakic, P. S. (1993). Dissociation of object and spatial processing domains in primate prefrontal cortex. Science, 260, 19551958.

Wojciulik, E., Kanwisher, N., \& Driver, J. (1998). Covert visual attention modulates face-specific activity in the human fusiform gyrus: fMRI study. Journal of Neurophysiology, 79, $1574-1578$.

Zeki, S., \& Marini, L. (1998). Three cortical stages of colour processing in the human brain. Brain, 121, 1669-1685. 